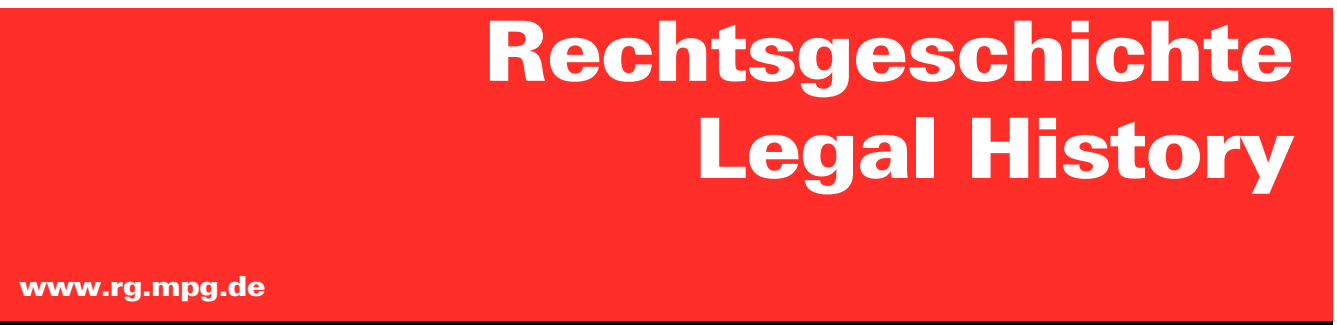

http://www.rg-rechtsgeschichte.de/rg26

$\operatorname{Rg} 26_{2018}$

$390-392$

Zitiervorschlag: Rechtsgeschichte - Legal History Rg 26 (2018)

http://dx.doi.org/10.12946/rg26/390-392

\title{
Caspar Ehlers*
}

\section{Integration durch Recht}

[Integration by Law]

* Max-Planck-Institut für europäische Rechtsgeschichte, Frankfurt am Main, ehlers@rg.mpg.de

Dieser Beitrag steht unter einer Creative Commons cc-by-nc-nd 3.0 
- Lounghis, Telemachos C., Basilike Blysidou, Stelios Lampakes (2005), Regesten der Kaiserurkunden des oströmischen Reiches von 476 bis 565 , Nicosia

- Mandouze, André (1982), Prosopographie de l'Afrique chrétienne (303-533), Paris

- Martindale, John R. (1980), The Prosopography of the Later Roman Empire II (A. D. 395-527), Cambridge

- Martindale, John R. (1992), The Prosopography of the Later Roman Empire III (A. D. 527-641), Cambridge

- Montinaro, Federico (2015), Les fausses donations de Constantin dans le Liber pontificalis, in: Millennium 12, 203-229

- Orlandis, José D., Domingo Ramos-Lissón (1981), Die Synoden auf der Iberischen Halbinsel bis zum Einbruch des Islam (711), Paderborn u. a.

- Pietri, Charles, Pietri, Luce (2000), Prosopographie chrétienne du Bas-Empire, II: Prosopographie de l'Italie chrétienne (313-604), I-II, Rom

- Pietri, Luce (2013), Prosopographie de la Gaule chrétiennne (314-614), I-II, Paris

- Riedinger, Rudolf (1988), Die Dokumente des Petrus notarius regionarius auf seiner Reise von Rom nach Spanien im Jahre $683 / 4$, in: Burgense 29, 233-250

- Riedinger, Rudolf (1998), Kleine Schriften zu den Konzilsakten des 7. Jahrhunderts (Instrumenta Patristica 34), Steenbrugge / Turnhout

- Scholz, Sebastian (2006), Politik - Selbstverständnis - Selbstdarstellung. Die Päpste in karolingischer und ottonischer Zeit (Historische Forschungen 26), Stuttgart

- Schwartz, Daniel R. (2015), New Light and Remaining Questions Concerning the Death of Philipp Jaffé, in: Concilium Medii Aevi 8, 61-80

- Schwartz, Daniel R. (2016), Between Jewish Posen and Scholarly Berlin. The Life and Letters of Philipp Jaffé, Berlin

- Seeck, Отto (1919), Regesten der Kaiser und Päpste für die Jahre 311 bis 476 n. Chr. Vorarbeit zu einer Prosopographie der christlichen Kaiserzeit, Stuttgart (Nachdr. Frankfurt am Main 1964)

- Strohm, Martin (1983), Der Konflikt zwischen Erzbischof Julian von Toledo und Papst Benedikt II.: Ein Faktum von ökumenischer Bedeutung, in: Annuarium historiae conciliorum 15, 249-259

- Winkelmann, Friedhelm (2001), Der monenergetisch-monotheletische Streit (Berliner Byzantinistische Studien 6), Frankfurt am Main u. a.

\section{Caspar Ehlers}

\section{Integration durch Recht*}

In seinem neuen Buch bietet Karl Ubl einen weit gefassten Überblick seiner bisherigen Forschungen zur Lex Salica im Kontext seines Projektes an der Universität Köln zu den frühmittelalterlichen Rechtsüberlieferungen, beginnend mit den substantiellen Beobachtungen »Warum Barbaren Gesetze erlassen « (37-66) als Folge aus der Frage nach Einsatz und Nutzen der Rechtsbücher (Einleitung, 11-35). Er sieht die fränkische Lex in ihren Ursprüngen als identitätsstiftendes Instrument an (»Ein Monument der Alterität«, 67-97, sowie als "Entwürfe von Gemeinschaft im 6. Jahrhundert", 99-135).

Die »Neufassung Pippins I.« bedeutete einen aus heutiger Sicht erkennbaren Eintritt in die schriftliche Überlieferung (137-163), die Neufas- sung Karls des Großen sogar eine Mystifizierung der Autorität des Rechts (165-191) vor der transformatorischen Phase des Untergangs des fränkischen Rechts (193-219) im Jahrhundert der Nachfolger Karls des Großen (zusammenfassend: „von der Lex Salica zu den Leges Francorum «, 218 f.). Das »Wissen über das Recht der Franken im 9. Jahrhundert « ist Gegenstand des achten Kapitels (221244).

Ausgehend von der Beobachtung, dass die älteste Fassung der Lex Salica wegen ihres archaischen Charakters kaum geeignet erscheint, das Rechtsbuch eines zur Großmacht aufsteigenden Reiches zu werden (11 ff.), referiert Ubl die Forschungskontroversen um die Deutung der Lex als »Urtext der deutschen Rechtsgeschichte« (13-24) und um 
die Ethnogenese der germanischen Völker (24-35). Geht es hier mehr um die wissenschaftliche Deutung bzw. Indienstnahme des Rechtsbuches für eine national orientierte Mediävistik, so wird in Abschnitt 2 die Frage in den Blick genommen, warum überhaupt »Barbaren Gesetze erlassen« und welche Vorstellungen räumlicher Geltung sie dabei leiteten - was Ubl anhand der schriftlichen Überlieferung sehr abgewogen problematisiert, da es Auswirkungen auf seine Datierungsvorschläge für die Genese der Frühfassungen der Lex hat (besonders 65 f.). Ging es den >barbarischen ‘ Franken bei der Niederschrift ihres Gesetzes um die Absonderung von >Rom< - oder wird dieser Eindruck durch die rechtshistorische Forschung zu den >Barbarenrechten< (beispielsweise durch Karl August Eckhardt) und besonders zu den 'Salfranken als Ethnon (negiert von Matthias Springer) erweckt?

Karl Ubl fährt mit seiner Analyse der Textstufen der Lex Salica fort (»Malbergische Glossen«, Geldstrafen etc.) und analysiert vor allem die ein Gericht betreffenden und die Anwendung der Lex rechtfertigenden Passagen (81-87). Er stellt darauf die Frage nach der Gesellschaft hinter der Lex Salica und dem »Bruch mit Rom«, den diese Gesetzgebung in einem Reich mit (einflussreichen) romanischen Bevölkerungsanteilen zur Folge gehabt haben muss. Welche »Entwürfe von Gemeinschaft im 6. Jahrhundert « (99-135) bilden die Basis für diese Fassungen der Lex Salica? Mehrere zeitgleich, so lautet die Antwort Ubls anhand der Analyse der chronologisch zu rekonstruierenden Überarbeitungen (103-133). Diese Dynamik der Anpassungen auf die Rechtswirklichkeit in sich unterscheidenden Geltungsräumen führte letztlich zu der Adaption des Rechtsmodells durch andere Gemeinschaften, wie den Alemannen im 7. Jahrhundert (siehe 135).

Da »die älteste Handschrift der Lex Salica [...] aus der Mitte des 8. Jahrhunderts und damit aus der Zeit Pippins des Jüngeren" stammt (137 ff.), fragt der Verfasser nach der Unsichtbarkeit des Rechtsbuches, die nur durch eine historiographische Quelle, den »Liber Historiae Francorum«, ein Schlaglicht bekommt, und konstatiert eine »Verformung des Rechts« durch König Pippin, mithin eine karolingische Transformation vor dem Hintergrund des geschwächten Königtums der Merowinger einerseits und des Konfliktes mit seinem Bruder Karlmann andererseits (144-148). Diese »DFassung « sei zwar als »Fehlschlag« (154) einzustufen, hätte aber den politischen Zielen Pippins als Ablöser der Merowinger und Bewahrer der rechtlichen Kontinuität dienen sollen (155-163).

Der ordnende Wille Karls des Großen ist nicht nur auf dem Gebiet des Rechts unumstritten erkennbar (165 ff.). Seine "Hinwendung zum Schriftrecht" zeichnet ihn aber besonders aus (167-174) und die intendierte Breitenwirkung der Rechtsreform (174-181) mittels der »Verschränkung von lex und capitulum « (181-186) wertet Karl Ubl als Beleg für den »Eigensinn der Franken« gegenüber den inzwischen unterworfenen Gebieten der Friesen und Sachsen (186 ff.) und zugleich als Versuch Karls des Großen, ein ursprünglich ethnisches Recht zu überhöhen (188191).

Bedeutete das 9. Jahrhundert den Höhepunkt der Überlieferung der Lex Salica, so sei dieser Zeitrahmen zugleich als »Untergang des fränkischen Rechts « $\mathrm{zu}$ bewerten, formuliert Karl Ubl (193 f.). Die Zeit Ludwigs des Frommen ließ die Lex Salica zu einem Recht der Eliten werden (195-205), das dem Römischen Recht widersprach (205-212). Karl der Kahle (reg. 843-877) hingegen habe den "Abschied vom gentilen Recht « eingeleitet und den Übergang "von der Lex Salica zu den Leges Francorum « eröffnet (212-219). Das »Wissen über das Recht der Franken im 9. Jahrhundert" beendet den analytischen Teil des zu besprechenden Buches (221-244) und summiert die zuvor gewonnenen Ergebnisse.

Ambitioniert kommt dann als Schluss das neunte Kapitel einher: »Für eine andere Rechtsgeschichte« (245-254). Ubl setzt sich mit der zementiert erscheinenden Forschungsmeinung auseinander, es sei erst im 12. Jahrhundert mit der Wiederentdeckung des Römischen Rechts und dessen gelehrter Form in einer Art »Urknall« aus dem Nichts (des Frühmittelalters) eine Rechtsgeschichte, die diesen Namen verdiene, entstanden. Vor allem Harold Bermans griffige Formulierung von einer "Revolution des Rechts « ${ }^{1}$ habe dazu maßgeblich

1 Harold Joseph Berman, Law and

Revolution. The Formation of the

Western Legal Tradition, Cam-

bridge / Mass. 1983. 
beigetragen, während die deutsche Rechtsgeschichte sich stets der nicht-römischen Rechte (»germanistisches Paradigma«) bedient habe (245 f.). Der Verfasser möchte ausdrücklich nicht gegen die These vom revolutionären Charakter des 12. Jahrhunderts vorgehen, sondern unter Berufung auf Maurizio Lupoi ${ }^{2}$ und über diesen hinausgehend die Bedeutung des Bruchs, »den die Lex Salica in der Entwicklung des Rechts im Frankenreich herbeiführte« (247), hervorheben, weil mit ihrer Hilfe gentile, weltliches Recht betreffende Rechtsordnungen auf andere Völker, beispielsweise die Sachsen, übertragen werden konnten (247 ff.). Mithin stellte sie ein bedeutendes Mittel zur Integration dieses expandierenden Reiches dar. Nicht zuletzt die überlieferten Exemplare des Rechtsbuches aus dem 9. und vor allem dem 10. Jahrhundert belegen diese Relevanz (250 f.), weshalb Karl Ubl dafür plädiert, weder von einer Revolution zu sprechen noch von einer Evolution im Sinne einer rechtshistorischen Kontinuitätslehre. Vielmehr sei die Bedeutung der Lex Salica auf einem anderen Feld zu sehen: dem der ethnischen Identitätsbildung, hier lägen die »Sinnstiftungen eines Rechtsbuchs«.

Zwei Bemerkungen seien dazu dem Rezensenten gestattet. Allgemeiner wäre der Hinweis darauf, dass es eine voraussetzungsfreie Revolution strictu senso gar nicht geben kann, was in den internationalen geschichtswissenschaftlichen Studien zu dem Phänomen einer umfassenden Umwälzung der Lebensordnungen und -wirklichkeiten im 12. Jahrhundert, die Ubl verständlicherweise nicht anführt, inzwischen auch communis opinio ist. Die zweite bezieht sich auf das Buch Ubls, das sich fragen lassen muss, welche Rolle die Kirche und ihre Rechtstraditionen bei den Genesen und Integrationen des früheren Mittelalters in der zweiten Hälfte des ersten Jahrtausends gespielt haben. Wäre die Integration Sachsens alleine mit dem Erlass der Lex Saxonum 802 oder 803 ein Erfolg gewesen?

\section{Caspar Ehlers \\ Rechtsräume der Kirche*}

Im vorvergangenen Jahr sind zwei umfangreiche Monographien zur Funktion der Kirche im Frühmittelalter erschienen, die besonderes Augenmerk unter der Fragestellung nach dem Verhältnis von »Recht, Raum und Religion« zu verdienen scheinen. Zum einen ist dies die Jenaer Dissertation von Tina Bode, zum anderen die Studie von Florian Mazel, Professor für mittelalterliche Geschichte an der Universität Rennes II, die Gegenstand seiner habilitation à diriger les recherches gewesen ist. Erstgenannte will sich auf das ostfränkische Reich der Ottonenzeit (919-1024) kon- zentrieren, während Mazel gar die mittelalterliche Kirche vom 5. bis zum 13. Jahrhundert in den Blick nehmen möchte.

Denn nichts weniger als die »mittelalterliche Erfindung des Raumes (5. bis 13. Jahrhundert)« verheißt der ambitionierte Untertitel des Buches von Florian Mazel, auch wenn diese Ankündigung inzwischen etwas verbraucht erscheint. Weil der Untersuchungsraum auf die Kirchenprovinzen Aix und Tours beschränkt wird (26-29), legen schon diese geographischen Schwerpunktsetzungen den Verdacht nahe, dass sich sein Buch kaum ernsthaft
2 Maurizio Lupoi, Alle radici del mondo giuridico europeo. Saggio storico-comparativo, Rom 1994; engl. Übersetzung: The Origins of the European Legal Order, transl. by Adrian Belton, Cambridge / Mass. 2000.

\author{
* Tina Bode, König und Bischof \\ in ottonischer Zeit: Herrschafts- \\ praxis, Handlungsspielräume, Inter- \\ aktionen (Historische Studien 506), \\ Husum: Matthiesen Verlag 2015, \\ ISBN 978-3-7868-1506-8; \\ Florian Mazel, L'Évêque et le
}

territoire. L'invention médiévale de l'espace (Ve-XIIIe siècle), Paris: Éditions du Seuil 2016, ISBN 978-2-02-118310-8 